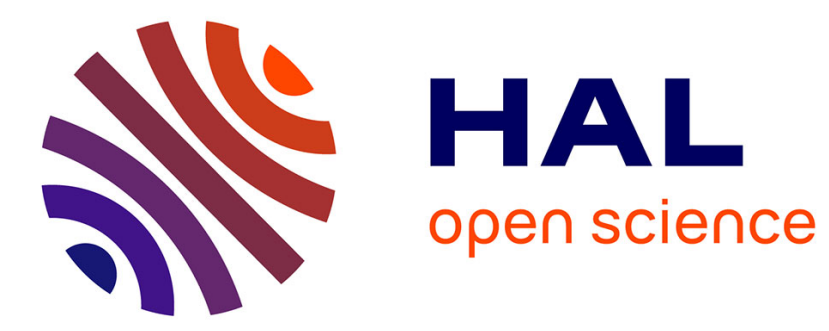

\title{
Experimental Validation of a New Dynamic Muscle Fatigue Model
}

Deep Seth, Damien Chablat, Sophie Sakka, Fouad Bennis

\section{To cite this version:}

Deep Seth, Damien Chablat, Sophie Sakka, Fouad Bennis. Experimental Validation of a New Dynamic Muscle Fatigue Model. 18th International Conference on Human-Computer Interaction, Jul 2016, Toronto, Canada. hal-01309155

\section{HAL Id: hal-01309155 https://hal.science/hal-01309155}

Submitted on 28 Apr 2016

HAL is a multi-disciplinary open access archive for the deposit and dissemination of scientific research documents, whether they are published or not. The documents may come from teaching and research institutions in France or abroad, or from public or private research centers.
L'archive ouverte pluridisciplinaire HAL, est destinée au dépôt et à la diffusion de documents scientifiques de niveau recherche, publiés ou non, émanant des établissements d'enseignement et de recherche français ou étrangers, des laboratoires publics ou privés. 


\title{
Experimental Validation of a New Dynamic Muscle Fatigue Model
}

\author{
Deep SETH, Damien CHABLAT, Sophie SAKKA, Fouad BENNIS \\ IRCCyN, Ecole Centrale de Nantes \\ 1, Rue de la Noë, Nantes, 44300, France \\ E-mail: Deep.Seth@irccyn.ec-nantes.fr, Damien.Chablat@irccyn.ec-nantes.fr, \\ Sophie.Sakka@irccyn.ec-nantes.fr, Fouad.Bennis@ec-nantes.fr
}

\begin{abstract}
Muscle fatigue is considered as one of the major risk factor causing Musculo-Skeletal Disorder (MSD). To avoid MSD the study of muscle fatigue is very important. For the study of muscle fatigue a new model is developed by modifying the Ruina Ma's dynamic muscle fatigue model and introducing the muscle co-contraction factor ' $n$ ' in this model.The aim of this paper is to experimentally validate a dynamic muscle fatigue model using Electromyography (EMG) and Maximum Voluntary Contraction (MVC) data. The data of ten subjects are used to analyze the muscle activities and muscle fatigue during the extension-flexion (push-pull) motion of the arm on a constant absolute value of the external load. The findings for co-contraction factor shows that the fatigue increases when cocontraction area decreases. The dynamic muscle fatigue model is validated using the MVC data, fatigue rate and co-contraction factor of the subjects.
\end{abstract}

Keywords: Muscle fatigue, maximum voluntary contraction (MVC), muscle fatigue model, co-contraction, fatigue rate, electromyography (EMG)

\section{Introduction}

In the field of industrial bio-mechanics, muscle fatigue is defined as "any reduction in the maximal capacity to generate the force and power output". In industries, mostly repetitive manual tasks leads to work related Musculo-Skeletal Disorder (MSD) problems $[1,2]$. Some times people have to work more on the same repetitive task which can be painful and leads to MSD due to muscle fatigue. MSD can cause pain $[1,3,4]$ or temporary dysfunction of the affected muscles [5, 6]. Muscle fatigue and uncomfortable working postures can cause drop in the productivity of human. To improve the performance and production, improvement in the work environment and ergonomics with the study of muscle fatigue are necessary that can reduce the chances of MSD [4].

Various static and dynamic muscle fatigue models are proposed earlier to study muscle fatigue [7, 8, 9, 10, 11, 12]. Liang's fatigue model [9] have experiment validation for fatigue and effect of recovery in arm with static drilling posture. Silva [13] simulate the hill model and validate it using opensim. Some Dynamic fatigue models are also introduced [14, 15, 16]. A Dynamic Muscle Fatigue Model [17] has been proposed to 
describe the fatigue process of muscle groups. However, no consideration about the cocontraction of paired muscles is taken. Missenard [18] explains the effect of fatigue and co-contraction on the accuracy of arms motion.

The main objective of this study is to revise this dynamic muscle fatigue model by including the factor of co-contraction of paired muscles, as well as to validate it through mathematics and experiments. In this article, we are focusing on the study of muscle activity with co-contraction, using elbow joint's muscle groups as target. With the assistance of EMG, the function of co-contraction is confirmed and calculated. Using the MVC data calculated during the fatigue test experiments, we have validated the muscle fatigue model.

\section{Dynamic muscle fatigue model}

The dynamic muscle fatigue model is applicable on the dynamic motion of the human body parts. The motions like push/pull operations of the arm, walking, pronation, supination etc. are examples of dynamic motion. A dynamic muscle fatigue model is proposed by Liang Ma $[9,19]$ firstly applied on static drilling task. Ruina Ma [16, 17] developed this model for the dynamic motions like push/pull operation of the arm. The Ruina Ma's model can be described by the equation 1 and 2. However, the cocontraction of the muscles are not included in both the models.

$$
\frac{d \Gamma_{c e m}(t)}{d t}=-k \frac{\Gamma_{\text {cem }}}{\Gamma_{M V C}} \Gamma_{\text {joint }}(t)
$$

and, if $\Gamma_{\text {Joint }}$ and $\Gamma_{M V C}$ held constant, the model can then simplify as follows:

$$
\Gamma_{\text {cem }}(t)=\Gamma_{M V C} \cdot \mathrm{e}^{-k_{\text {torque } C t}}, \quad \text { where } C=\frac{\Gamma_{\text {Joint }}}{\Gamma_{M V C}}
$$

The parameters for this model is expressed in the table 1

\begin{tabular}{|l|l|l|}
\hline Elements & Unit & Description \\
\hline$k$ & $\min ^{-1}$ & Fatigue factor, constant \\
$\Gamma_{M V C}$ & N.m & Maximum torque on joint \\
$\Gamma_{\text {Joint }}$ & N.m & Torque from external load \\
$\Gamma_{c e m}$ & N.m & Current capacity of the muscle \\
\hline
\end{tabular}

Table 1: Parameters of Ruina Ma's dynamic muscle fatigue model

\subsection{Hypothesis for New Dynamic Muscle Fatigue Model}

Muscle fatigue is directly proportional to the torque applied at the human joint. It is also inversely proportional to the maximum capacity (without fatigue) of muscle to generate a torque $\Gamma_{M V C}$ (Maximum MVC). According to this model, the evolution of $\Gamma_{c e m}$ (Current capacity of the muscle) can be represented by a linear differential equation of the first order. 
As we know, there are two major muscle groups for each joint motion, agonist and antagonist. For push motion, a muscle motivates the motion while antagonist muscle makes the motion accurate and stable. If the motion is reversed, i.e, pull cycles, agonist and antagonist muscles switch their roles. Co-operation of the two muscles is called co-contraction.

\subsection{Proposed dynamic model of muscular fatigue}

In dynamic muscle fatigue model [20], we select two parameters $\Gamma_{j o i n t}$ and $\Gamma_{M V C}$ to build our muscle fatigue model. The hypotheses can then be incorporated into a mathematical model of muscle fatigue which is expressed as follows:

$$
\frac{d \Gamma_{\text {cem }}(t)}{d t}=-k \cdot n \cdot \frac{\Gamma_{\text {cem }}}{\Gamma_{M V C}} \Gamma_{\text {joint }}(t)
$$

where, $k$ is the fatigue factor and $n$ is the co-contraction factor.

And, if $\Gamma_{\text {Joint }}$ and $\Gamma_{M V C}$ held constant, the model can then simplify as follows:

$$
\begin{gathered}
\Gamma_{c e m}(t)=\Gamma_{M V C} \cdot \mathrm{e}^{-k \cdot n \cdot C t}, \quad \text { where } C=\frac{\Gamma_{\text {Joint }}}{\Gamma_{M V C}} \\
k=\frac{-1}{n \cdot C t} \cdot \ln \left(\frac{\Gamma_{\text {cem }}(t)}{\Gamma_{M V C}}\right)
\end{gathered}
$$

The other parameters for this model are the same as in table 1 . We define $n$ as the co-contraction factor.

\subsection{Co-contraction factor ' $n$ '}

The co-contraction is the simultaneous contraction of both the agonist and antagonist muscle around a joint to hold a stable position at a time. Assumptions made for finding co-contraction factor are as follows:

1. The co-contraction is the common intersecting area between the two groups of acting muscles.

2. The co-contraction factor will be the same for each agonist and antagonist activities.

The co-contraction area can be understand by the figure 1. This figure is just an example representation of a motion cycle. In this figure, we can introduce the common EMG activity between bicep and tricep muscle groups shown by the orange color, which is co-contraction area $C_{A}$. The formula for calculating the co-contraction area from EMG activities is given in equation 6 . The trapezius activity shown along with the two muscles is co-activation.

$$
C_{A}=\frac{\int_{t_{0}}^{t_{100}} E M G_{\text {min }} \times d t}{\int_{t_{0}}^{t_{100}}\left[E M G_{\text {agonist }}+E M G_{\text {antagonist }}\right] \times d t}
$$

where, $E M G_{\min }$ is the common area share by the EMG activity of bicep and tricep, $E M G_{\text {agonist }}$ and $E M G_{\text {antagonist }}$ are the full activities of the bicep and triceps muscle's. 
The activities of the both the muscles are normalized with respect to the normalization value of the activities for the same muscle which can be calculated using the equation 10, it is because the absolute value of the external torque is the same for push/pull operation.

The co-contraction area $C_{A}$ can also be represented as follows:

$C_{A}=$ common activities between the two muscle groups.

$$
C_{A}=a \cdot \exp b \cdot x
$$

where, $a$ and $b$ are constant parameters and $x$ is the time of the test.

In our model, the co-contraction factor represents the main activities of muscle in each dynamic cycle excludes the co-contraction area of the same cycle. So we can represent co-contraction factor $n$ as follows:

$$
\begin{gathered}
n=1-C_{A} \\
n=1-a \cdot \exp b \cdot x
\end{gathered}
$$

\subsection{Push-Pull Operation and Muscles activities}

The push/pull motion of the arm is the flexion and extension of the arm about the elbow. The plane of the motion is vertical plane. The Push/pull activities with the muscle activation is shown in figure 3. In Ma's model there were no part of co-contraction and delay in the model which we have added in this new model.
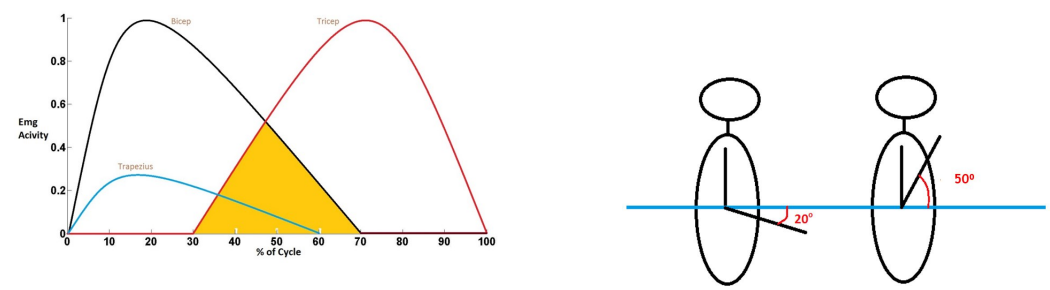

Fig. 1: A representative plot of EMG activity of bicep, triceps and trapezius normalized with the maximum value of Fig. 2: Arm movement range while each muscle's activity for one cycle

\section{Methodology: Experiment and Data Processing}

\subsection{Experiment protocol}

1. The repetitive arm's flexion-extension in a vertical plane as shown in figure 2.

2. The motion range is seventy degrees. The test protocol repetition continues till exhaustion.

3. Each cycle (flexion + extension) should be completed within 3 seconds.

4. External load was $20 \%$ of MVC. MVC was calculated every one minute. 


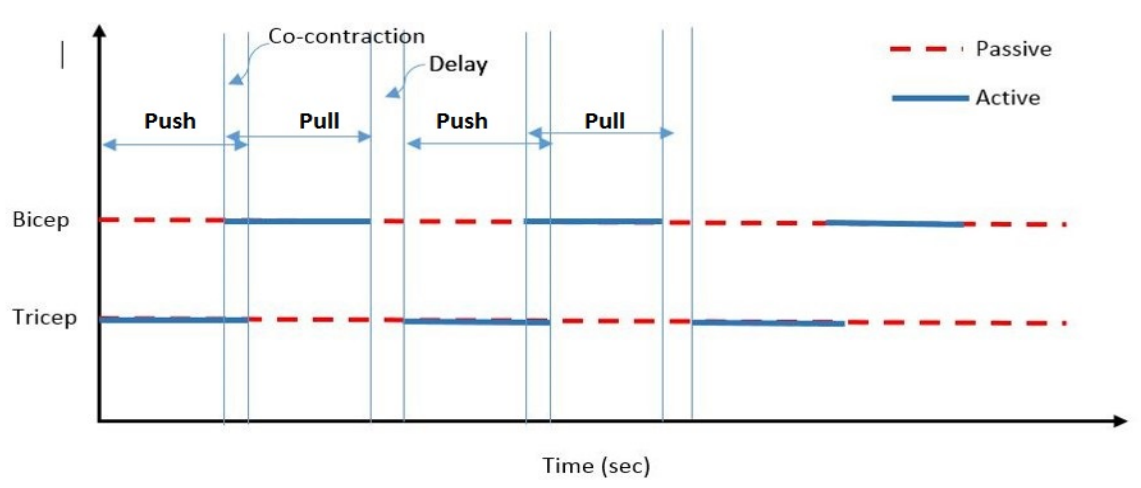

Fig. 3: Push/Pull Motion and Muscles activities

\subsection{Data Acquisition}

A Biodex system 3 research (Biodex medical,shirley, NY) isokinetic dynamo-meter was used to measure the value of elbow angle, velocity and torque. The Electromyographic sensor electrodes were put on Biceps, Tricep and Trapezius muscles to record their electrical activities. The frequency of data acquisition was set at $2000 \mathrm{~Hz}$.

\subsection{Subjects description}

The subjects (all male) details are given in the table 2. All the subjects were sportive. The subjects were physically fit and had no injuries in the upper limb.

\begin{tabular}{|l|l|l|c|c|c|c|}
\hline Subject & Age & Weight & Height & Upper arm & Forearm & Sports \\
\hline \hline 1 & 28 & $89 \mathrm{~kg}$ & $185 \mathrm{~cm}$ & $29 \mathrm{~cm}$ & $26.5 \mathrm{~cm}$ & Running \\
2 & 24 & $80.2 \mathrm{~kg}$ & $183.5 \mathrm{~cm}$ & $31.5 \mathrm{~cm}$ & $28 \mathrm{~cm}$ & Musculation \\
3 & 20 & $69.8 \mathrm{~kg}$ & $180.1 \mathrm{~cm}$ & $30 \mathrm{~cm}$ & $29.5 \mathrm{~cm}$ & Handball \\
4 & 20 & $80.9 \mathrm{~kg}$ & $177 \mathrm{~cm}$ & $29.8 \mathrm{~cm}$ & $29 \mathrm{~cm}$ & Handball \\
5 & 21 & $62.2 \mathrm{~kg}$ & $172.8 \mathrm{~cm}$ & $29.2 \mathrm{~cm}$ & $26.5 \mathrm{~cm}$ & Tennis \\
6 & 25 & $61.1 \mathrm{~kg}$ & $164.8 \mathrm{~cm}$ & $26 \mathrm{~cm}$ & $24.5 \mathrm{~cm}$ & Rugby \\
7 & 26 & $74 \mathrm{~kg}$ & $176 \mathrm{~cm}$ & $28.5 \mathrm{~cm}$ & $27 \mathrm{~cm}$ & Tennis \\
8 & 27 & $66 \mathrm{~kg}$ & $181 \mathrm{~cm}$ & $29.5 \mathrm{~cm}$ & $26.5 \mathrm{~cm}$ & wall climb \\
9 & 23 & $66.3 \mathrm{~kg}$ & $164 \mathrm{~cm}$ & $27 \mathrm{~cm}$ & $25.5 \mathrm{~cm}$ & Swimming \\
10 & 26 & $85 \mathrm{~kg}$ & $184 \mathrm{~cm}$ & $29 \mathrm{~cm}$ & $26.5 \mathrm{~cm}$ & Football \\
\hline
\end{tabular}

Table 2: Subjects anthropometric data and description 


\subsection{Data Processing and analysis}

All the raw data were processed using standardized MATLAB program. Data processing includes noise filtering from raw EMG data with the filter frequency $10 \mathrm{~Hz}$ for low pass filter and $400 \mathrm{~Hz}$ for high pass filter and normalization of the data. The total number of cycles compared for all the ten subjects are 1998 cycles. All the cycles are normalized on time scale and compared. The cycle selection for flexion and extension phases is done according to the velocity change in each cycle. The collective EMG plots for Biceps, Triceps and Trapezius muscle are show in figure $4 \mathrm{a}$ and figure $4 \mathrm{~b}$ for all the ten subjects and the collective comparison for the mechanical data position, velocity and torque is shown in figure $5 \mathrm{~b}$ and figure $5 \mathrm{a}$ for all the ten subjects.

For figure $4 a, 4 b, 5 a a n d 5 b$ representations are as follows:

--- Blue color curve show mean EMG activity.

I Red bar plot on blue curve shows the standard deviation of all the EMG activities along the mean.

- Black dotted curves shows the maximum and minimum reach from the EMG activies. All the cycles are normalized according to the equation:

$$
\text { value }_{\text {Normalization }}=\text { value }_{\text {std }} \text { max }+2 \sigma
$$

- value Normalization : Normalization value for the EMG data.

- value std $^{\max }$ : Maximum value of standard deviation along the mean.

$-2 \sigma: \sigma$ values addition upto $2 \sigma$

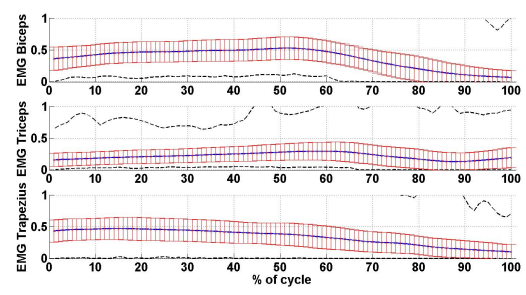

(a) Flexion/pull phase for all the subjects

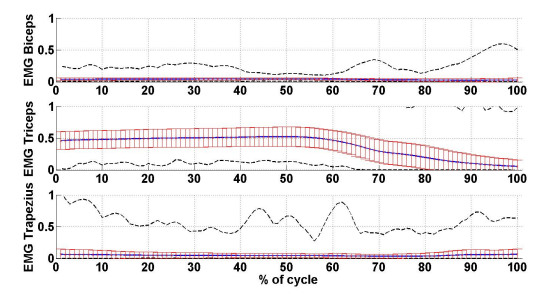

(b) Extension/push Phase for all the subjects

Fig. 4: Mean and Standard deviation plots for EMG data of Bicep, Triceps and Trapezius

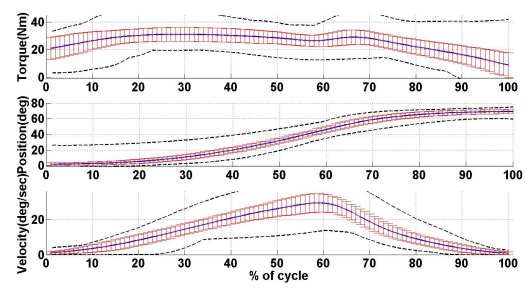

(a) Flexion/pull Phase for all the subjects

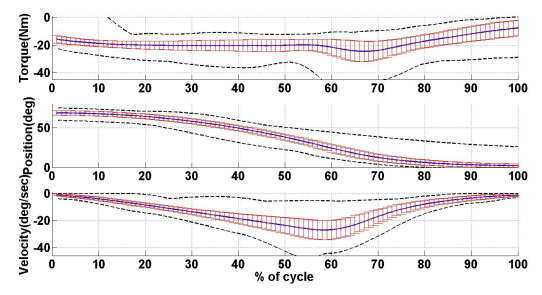

(b) Extension/push Phase for all the subjects

Fig. 5: Mean and Standard deviation plots for velocity, position and torque 


\section{Results and discussion}

The raw data obtained after the fatigue test is processed and the results and observations are discussed in this section. After processing the EMG data of all the muscle groups from figure 4 and 5 we can observe that when the biceps are active during flexion phase there are always some activities from the triceps and on the other hand when triceps are active during pull phase the biceps are almost passive or activities are very near to zero. We can also observe the co-activation of trapezius muscle with the activation of biceps. The activation of triceps with the biceps is co-contraction between two muscles during flexion phase.

The co-contraction area calculated by using equation 6 is fitted with the exponential equation 7 in section 2.3. The figure 6 shows the fitted graphs for the co-contraction percentage for test cycles of all ten subjects. In figure 6 blue dots show the percentage area of contraction during each extension-flexion cycle and red curve shows the exponential fit for the percentage co-contraction. This shows that the co-contraction percentage for activity between the muscles reduces as the fatigue test proceed or the muscles gets fatigued. By the equation 6 and 3 we can find $n_{i}$ as shown in table 3, where $i$ is the subject number:

\begin{tabular}{|c|c|c|c|c|c|c|c|c|c|c|}
\hline$n_{i}$ & $n_{1}$ & $n_{2}$ & $n_{3}$ & $n_{4}$ & $n_{5}$ & $n_{6}$ & $n_{7}$ & $n_{8}$ & $n_{9}$ & $n_{10}$ \\
\hline \hline Mean co-contraction factor & 0.6 & 0.55 & 0.67 & 0.6 & 0.59 & 0.65 & 0.64 & 0.74 & 0.5 & 0.7 \\
\hline
\end{tabular}

Table 3: Co-contraction factor for each subject

We can notice that only the subject number 8 in figure 6 h has increasing slope for the co-contraction area. This behavior can be associated with his sport activity which is wall climbing and very different from other subjects as shown in table 2 .

The co-activation of the trapezius muscle is observed mostly in the flexion phase. The MVC values are measured between each protocol of one minute. In figure 7 blue line shows the MVC measured for flexion and extension after each test protocol of 1 minute. We can see in most of the cases MVC decreases as fatigue increases. The MVC is same as $\Gamma_{\text {cem }}$ used in our model. The theoretical and experimental evolution of $\Gamma_{\text {cem }}$ is on the basis of $k$ (fatigue rate) using equation 4 and equation 4 and calculated $n_{i}$ and $C=0.2$. The evaluation of fatigue parameter ' $k$ ' for $\Gamma_{\text {cem }}$ extension is shown in figure $7 \mathrm{a}, 7 \mathrm{c}, 7 \mathrm{e}, 7 \mathrm{~g}, 7 \mathrm{i}, 7 \mathrm{k}, 7 \mathrm{~m}, 7 \mathrm{o}, 7 \mathrm{q}$ and $7 \mathrm{~s}$. Similarly fatigue parameter ' $k$ ' evaluation for $\Gamma_{\text {cem }}$ flexion is shown in figure $7 \mathrm{~b}, 7 \mathrm{~d}, 7 \mathrm{f}, 7 \mathrm{~h}, 7 \mathrm{j}, 7 \mathrm{l}, 7 \mathrm{n}, 7 \mathrm{p}, 7 \mathrm{r}$ and $7 \mathrm{t}$. The theoretical and experimental evolution of $\Gamma_{\text {cem }}$ shows that the experimental values are well fit with in the theoretical model. The co-contraction factor have significant effect on the model. The fatigue rate increases with the input of co-contraction factor. The minimum, maximum and average value of ' $k$ ' for each subject are shown in table 4 . 


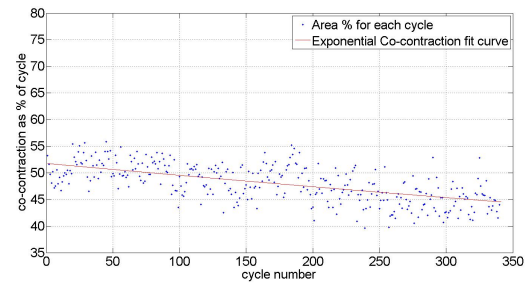

(a) subject 1

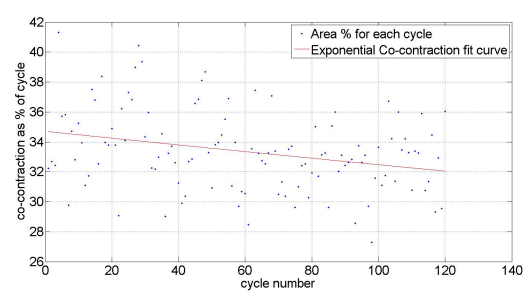

(c) subject 3

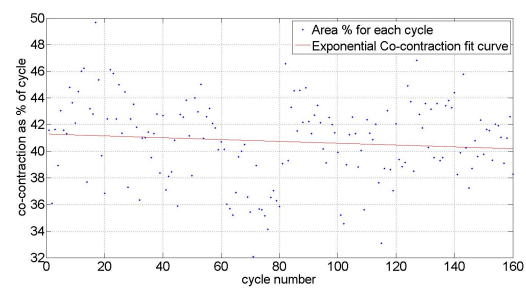

(e) subject 5

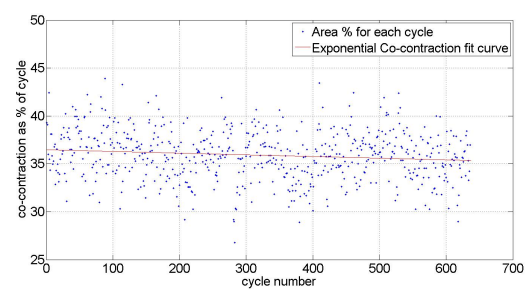

(g) subject 7

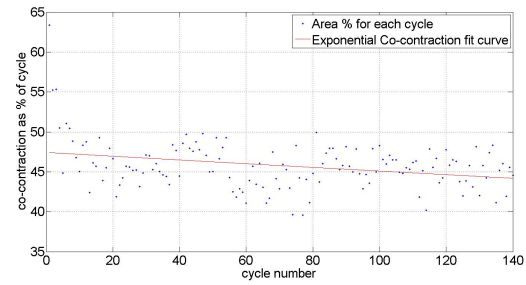

(i) subject 9

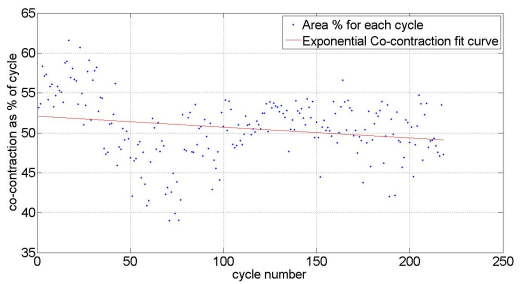

(b) subject 2

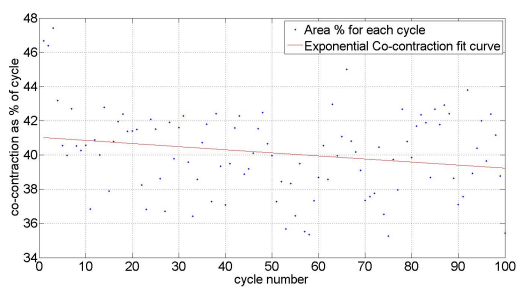

(d) subject 4

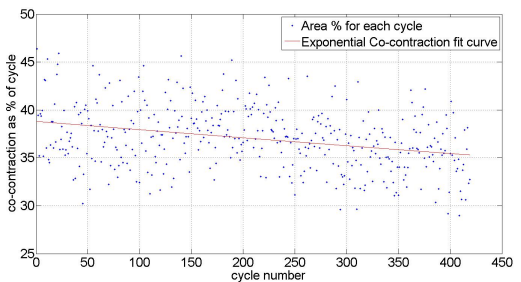

(f) subject 6

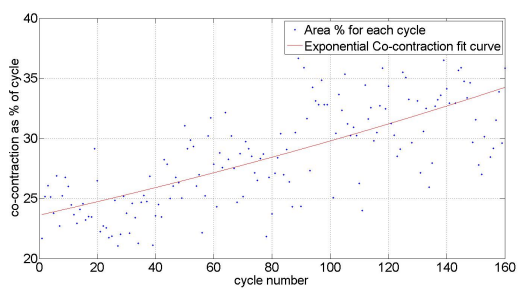

(h) subject 8

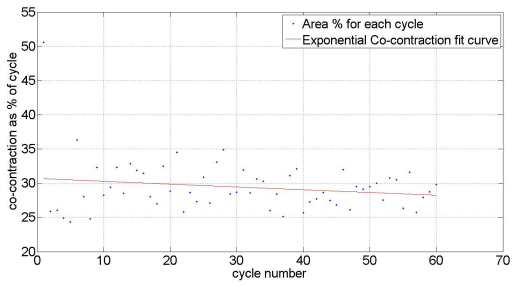

(j) subject 10

Fig. 6: Exponential curve fit for the co-contraction area 


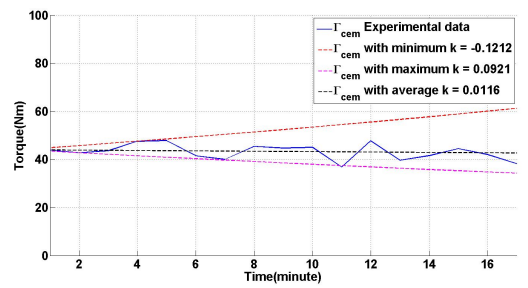

(a) The extension in the subject 1

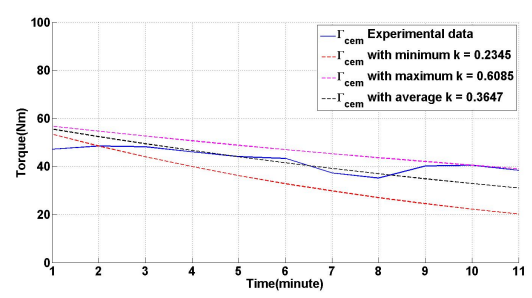

(c) The extension in the subject 2

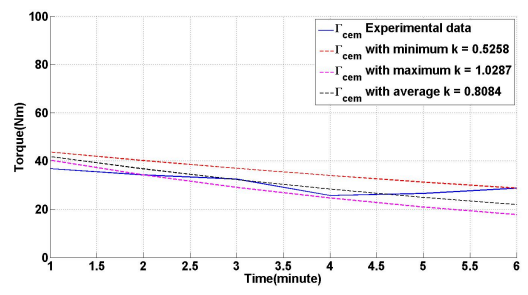

(e) The extension in the subject 3

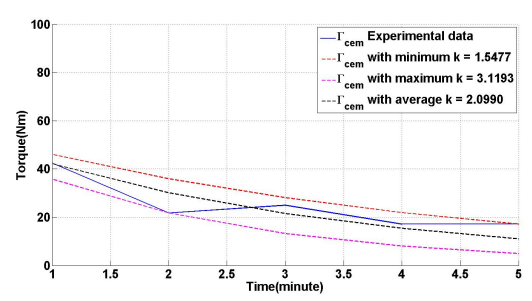

(g) The extension in the subject 4

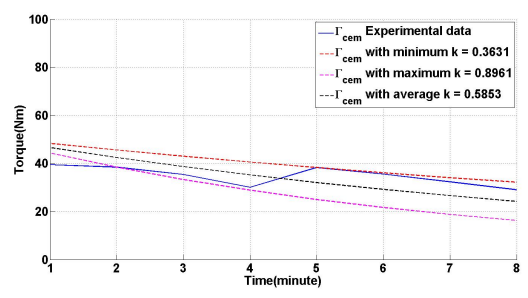

(i) The extension in the subject 5

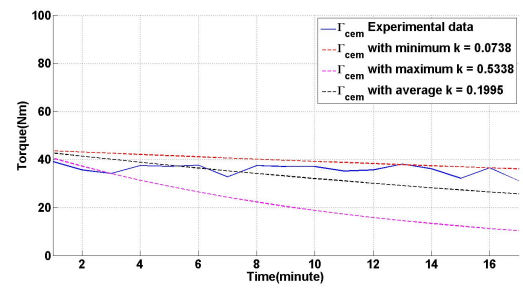

(b) The flexion in the subject 1

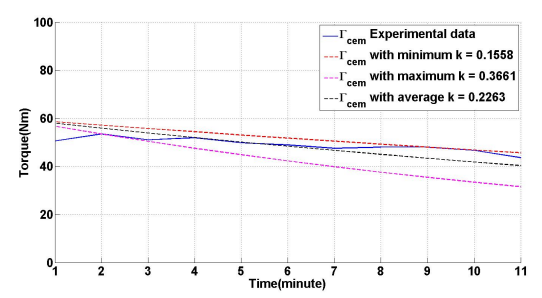

(d) The flexion in the subject 2

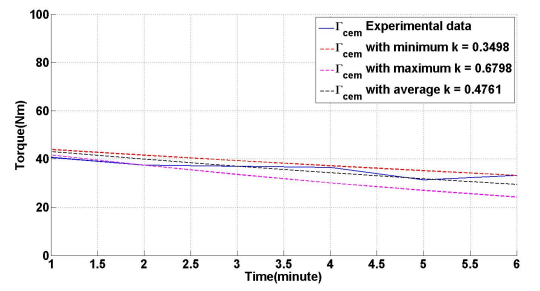

(f) The flexion in the subject 3

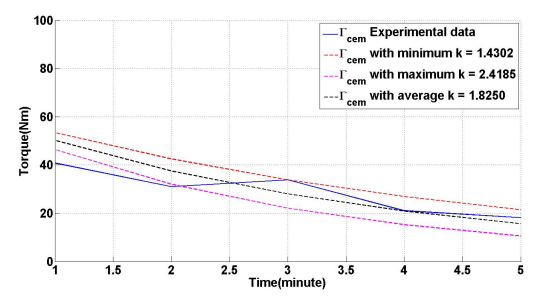

(h) The flexion in the subject 4

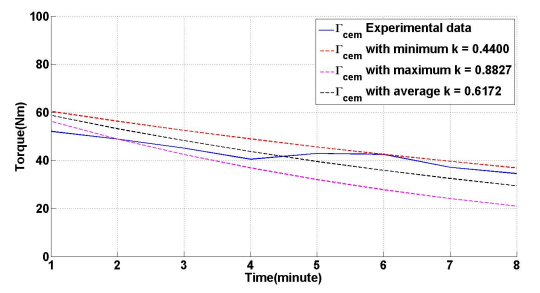

(j) The flexion in the subject 5 


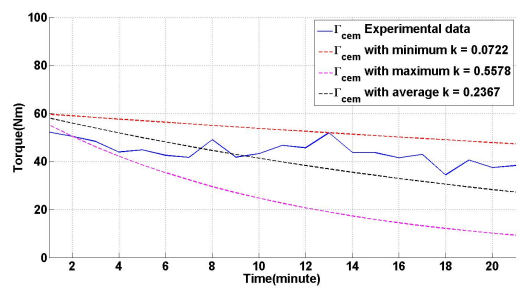

(k) The extension in the subject 6

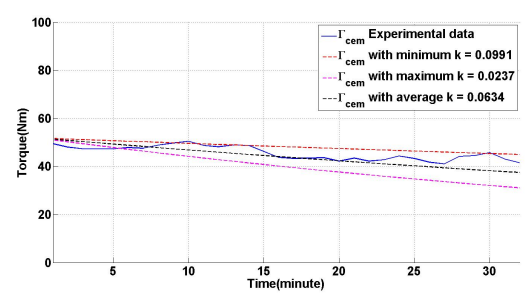

(m) The extension in the subject 7

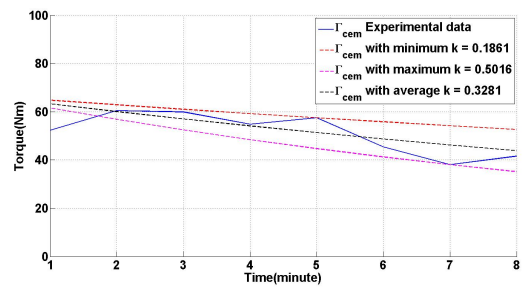

(o) The extension in the subject 8

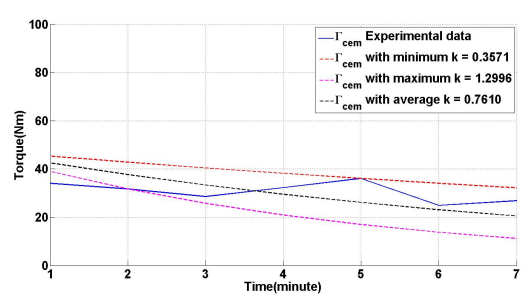

(q) The extension in the subject 9

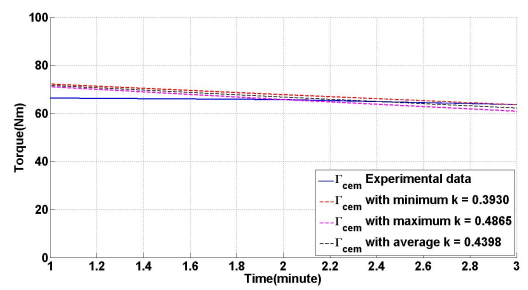

(s) The extension in the subject 10

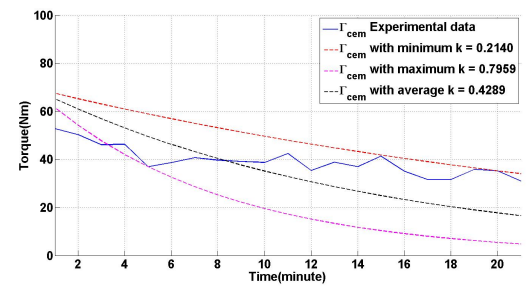

(1) The flexion in the subject 6

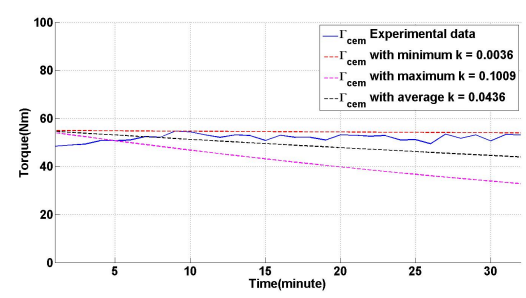

(n) The flexion in the subject 7

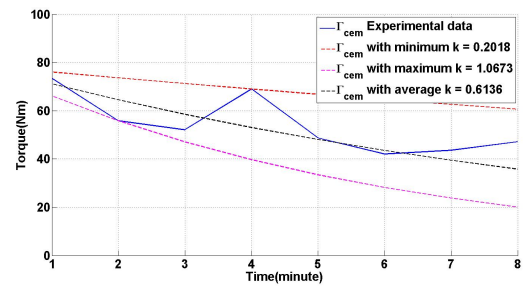

(p) The flexion in the subject 8

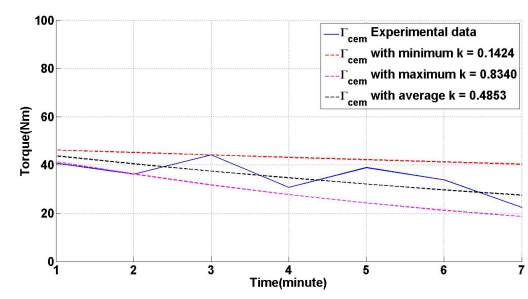

(r) The flexion in the subject 9

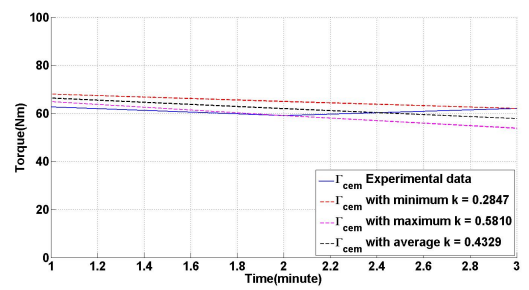

(t) The flexion in the subject 10

Fig. 7: Theoretical evolution of $\Gamma_{\text {cem }}$ and experimental data using different values of $k$ 


\begin{tabular}{|c||c|c|c|c|c|c|}
\hline & \multicolumn{3}{|c|}{$k_{\text {extension }}$} & \multicolumn{3}{c|}{$k_{\text {flexion }}$} \\
\hline Subject number & Minimum & Maximum & Average & Minimum & Maximum & Average \\
\hline 1 & -0.1212 & 0.0921 & 0.0116 & 0.0738 & 0.5338 & 0.1995 \\
2 & 0.2345 & 0.6085 & 0.3647 & 0.1558 & 0.3661 & 0.2263 \\
3 & 0.5258 & 1.0287 & 0.8084 & 0.3498 & 0.6798 & 0.4761 \\
4 & 1.5477 & 3.1993 & 2.0990 & 1.4302 & 2.4185 & 1.8250 \\
5 & 0.3631 & 0.8961 & 0.5853 & 0.4400 & 0.8827 & 0.6172 \\
6 & 0.0722 & 0.5578 & 0.2367 & 0.2140 & 0.7959 & 0.4289 \\
7 & 0.0237 & 0.0991 & 0.0634 & 0.0036 & 0.1009 & 0.0436 \\
8 & 0.1861 & 0.5061 & 0.3281 & 0.2018 & 1.0673 & 0.6136 \\
9 & 0.3571 & 1.2996 & 0.7610 & 0.1424 & 0.8340 & 0.4853 \\
10 & 0.3930 & 0.4865 & 0.4398 & 0.2847 & 0.5810 & 0.4329 \\
\hline
\end{tabular}

Table 4: Experimentally calculated values of ' $k$ ' for flexion and extension motion

\section{Conclusions}

The proposed model for dynamic muscle fatigue includes the co-contraction parameter, unlike in any other existing model according to the author's knowledge. The results and analysis of the experimental data validates the most of the assumptions made for the proposed model. EMG analysis along with MVC helps to understand the muscle activities, it justifies the significance of the co-contraction parameter in proposed dynamic muscle fatigue model. The experimental data also helps in validating the new dynamic muscle fatigue model.

\section{Acknowledgments}

The project is funded by the European Commission under the 'Erasmus Mundus Heritage Program', jointly coordinated by Ecole Centrale de Nantes, France and Indian institute of Technology Madras, India. The Experiments was performed in STAPS, University of Nantes, France. We are also thankful to Antoine Nordez and Marc Jubeau from UFR STAPS, France for assistance in preparing and conducting the experiments.

\section{References}

[1] Nurhayati Mohd Nur, Siti Zawiah Md Dawal, and Mahidzal Dahari. The prevelence of work related musculosceletal disorders among workers performing industrial repetitive tasks in the automotive manufacturing companies. In Proceedings of the 2014 International conference on inductrial engineering and operations management, Bali, Indonesia, 2014.

[2] Laura Punnett and David H. Wegman. Work related musculoskeletal disorders: the epidemiologic evidence and the debate. Journal of Electromyography and Kinesiology, 14:1323, 2004.

[3] Angelika Huppe, Kristin Muller, and Heiner Raspe. Is the occurrence of back pain in germany decreasing? two regional postal surveys a decade apart. European Journal of Public Health, 17:318-322, 2006. 
[4] RC Lawrence, CG Helmick, FC Arnett, RA Deyo, DT Felson, EH Giannini, SP Heyse, R Hirsch, MC Hochberg, GC Hunder, MH Liang, SR Pillemer, VD Steen, and F Wolfe. Estimates of the prevalence of arthritis and selected musculoskeletal disorders in the united states. Arthritis \& Rheumatism, 41(5):778-799, 1998.

[5] World Health Organization. Preventing musculoskeletal disorders in the workplace. Protecting workers 'Health series; no. 5', Geneva 27, Switzeland, 2003. ISBN 924159053 $\mathrm{X}$.

[6] Don B Chaffin, Gunnar B J Andersson, and Bernard J Martin. Occupational Biomechanics. Wiley - Interscience, third edition, 1999.

[7] J Ding, AS Wexler, and SA Binder-Macleod. Mathematical models for fatigue minimization during functional electrical simulation. journal of electromyograpgy and kinesiology, 13:575-588, 2003.

[8] A.V. Hill. The heat of shortening and dynamic constant of muscle. Proc. R. Soc. Lond. B, 126:135-195, 1938.

[9] Liang Ma, Damien Chablat, Fouad Bennis, and W Zhang. A new muscle fatigue and recovery model and its ergonomics application in human simulation. Virtual and Physical Prototyping, 5:123-137, 2008.

[10] Elena V. Galiamova Vladimir V. Syuzev, Alexander M. Gouskov. Human skeletal muscle mechanical and mathematical models. ICABB-2010, Venice, Italy, 0:-, 2010.

[11] Vollestad and Nina K. Measurement of human muscle fatigue. Journal of Neuroscience Methods, 74:219-227, 1997.

[12] Ting Xia and Laura A. Frey Lawa. A theoretical approach for modeling peripheral muscle fatigue and recovery. Journal of Biomechanics 41 (2008) 30463052, 41:3046-3052, 2008.

[13] Jorge M. Martins Miguel T. Silva, Andre F. Pereira. An efficient muscle fatigue model for forward and inverse dynamic analysis of human movements. Procedia IUTAM, 2:262-274, 2011.

[14] Jouni Freund and Esa-Pekka Takala. A dynamic model of the forearm including fatigue. Journal of Biomechanics, 34:597-605, 2001.

[15] Jing Z. Liu, Robert W. Brown, and Guang H. Yue. A dynamical model of muscle activation, fatigue and recovery. Biophysical Journal, 82:2344-2359, 2002.

[16] Ruina Ma, Damien Chablat, and Fouad Bennis. Human muscle fatigue model in dynamic motions. Latest Advances in Robot Kinematics, 0:349-356, 2012.

[17] Ruina Ma, Damien Chablat, Fouad Bennis, and Liang Ma. A framework of motion capture system based human behaviours simulation for ergonomic analysis. In HCI International 2011, 9-14 July, Hilton Orlando Bonnet Creek, Orlando, Florida, USA, 2011.

[18] Olivier Missenard, Denis Mottet, and Stephane Perrey. Muscular fatigue increases signaldependent noise during isometric force production. Neuroscience Letters, 437:154157, 2008.

[19] Liang Ma, Damien Chablat, Fouad Bennis, and W Zhang. A new simple dynamic muscle fatigue model and its validation. International Journal of Industrial Ergonomics, 39(1):211-220, 2009.

[20] Ruina Ma, Damien Chablat, and Fouad Bennis. A new approach to muscle fatigue evaluation for push/pull task. In 19th CISM-IFToMM Symposium on Robot Design, Dynamics, and Control, Paris, France, pages 1-8, 2012. 